Title : will be set by the publisher

Editors : will be set by the publisher

EAS Publications Series, Vol. ?, 2004

\title{
EFFECTS OF ATMOSPHERIC TURBULENCE ON THE GENIE NULLING INTERFEROMETER
}

\author{
O. Absil ${ }^{1}$
}

\begin{abstract}
Two competitive design studies for the Ground-based European Nulling Interferometer Experiment (GENIE) have recently been initiated by the European Space Agency and the European Southern Observatory. A major issue in these studies is the influence of atmospheric turbulence on the performance of the instrument, and how atmospheric effects can be compensated in order to reach the goal performance (detection of faint exozodiacal clouds). In this paper, we review the main atmospheric processes affecting a nulling interferometer and discuss possible ways to reduce them by means of real-time control systems. Preliminary performance estimates of GENIE are then presented. The effects of the thermal background and its fluctuations (Absil \& Bakker 2004) are not considered here.
\end{abstract}

\section{Introduction}

In the context of the Darwin Technical Research Programme, the European Space Agency has initiated a definition study for a ground-based nulling interferometer, with the goal to test some of the key components and techniques of the Darwin space mission (including achromatic phase shifters, mid-IR single-mode fibers, delay lines, fringe sensors, detectors, cryogeny, ...). GENIE will consist in a Bracewell interferometer formed of two VLT 8-m Unit Telescopes (UT) or auxiliary 1.8-m telescopes (AT) operating in the infrared L' band $(3.5-4.1 \mu \mathrm{m}$, see Gondoin et al. 2004). It will validated on-ground the concept of nulling interferometry and allow the European scientific community to build experience in the operation of a nulling interferometer and in its specific data reduction. Besides its technical goals, GENIE will also prepare the Darwin science programme by surveying nearby main-sequence stars in order to detect exozodiacal dust clouds at the level of 20 times our local zodiacal cloud. This will allow to screen out the stars surrounded by too much dust, for which the exozodiacal emission becomes

\footnotetext{
${ }^{1}$ IAGL, University of Liège, B5C, 17 Allée du 6 Août, B-4000 Sart-Tilman, Belgium
} 


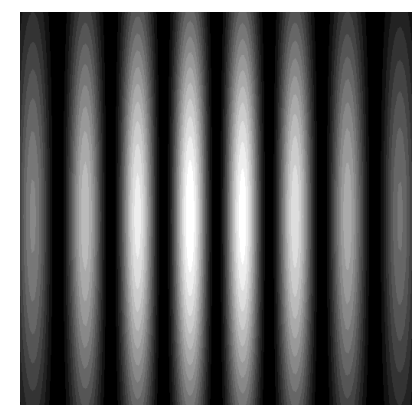

Fig. 1. Transmission map in the L' band for a $50 \mathrm{~m}$ Bracewell interferometer formed of two 8-m telescopes. The interferometric field-of-view is 125 mas while the spacing between two successive bright fringes is 17 mas.

the main source of noise in the case of Darwin. These dust disks are typically $10^{4}$ to $2 \times 10^{4}$ times fainter than their parent star in the L' band. In order to detect such faint disks with a high enough signal-to-noise ratio, GENIE needs to achieve calibrated nulling ratios of about $10^{5}$.

GENIE also aims at characterizing the inner part of protoplanetary disks around young stars, where planet formation could be detected by the presence of gaps or disk asymmetries. An additional objective of GENIE is to detect the thermal emission from a few bright hot Jupiters and to perform low-resolution spectroscopy to characterize their atmosphere.

\section{Principle of nulling interferometry}

The principle of nulling interferometry is to combine the light collected by two or more telescopes in a co-axial mode, adjusting their respective phase-shifts in order to produce a totally destructive interference on the optical axis (Bracewell 1978). The interferometer is characterized by its transmission map, displayed in Fig. 1 for a two-telescope interferometer, in one arm of which a phase shift of $\pi$ radians has been introduced (Bracewell interferometer). Because recombination is done in a pupil plane, no image is formed: the flux is integrated on the field-of-view and detected on a single pixel. The transmission map shows the parts of the field-ofview that are transmitted and those that are blocked by the interference process.

Even when the star is perfectly centered on the optical axis, a part of the stellar light still leaks through the transmission map due to the finite extent of the stellar disk. We define the rejection rate $\rho$ as the ratio between the initial stellar flux at the input of the beam-combiner and the stellar flux transmitted by the nuller at the destructive output. Assuming that the stellar angular radius $\theta_{*}$ is small as compared to the fringe spacing $\lambda / B$, and computing the transmitted flux as a two-dimension integral of the transmission map on the stellar disk, one gets the 
following expression for the rejection rate:

$$
\rho=\frac{4}{\pi^{2}}\left(\frac{\lambda}{B \theta_{*}}\right)^{2} .
$$

It is the fundamental figure of merit for a nulling interferometer. A typical value of the rejection rate for an L'-band Bracewell interferometer observing a Sun-like star at $10 \mathrm{pc}$ with a $50 \mathrm{~m}$ baseline is $\rho \simeq 460$.

The expression (2.1) of the rejection rate is valid only for a perfect Bracewell interferometer. In practice, the rejection rate is degraded by imperfect co-phasing of the light beams, intensity mismatches and polarization errors (Ollivier 1999). The residual stellar signal at the output of the interferometer can thus be divided into two parts:

- The geometric leakage, proportional to $1 / \rho$, is associated to the finite size of the stellar disk and depends on the length of the interferometer baseline. Calibration techniques such as precise measurements of stellar diameters can be used to reduce this contribution down to an acceptable level $\left(10^{-5}\right.$ of the initial stellar flux).

- The instrumental leakage, produced by the effects of atmospheric turbulence and by various instrumental errors, will be discussed in more detail in the next section. This contribution does not depend on the stellar angular size to the first order.

\section{Effects of atmospheric turbulence on a nulling interferometer}

\subsection{Effects of phase errors}

The main contributors to the differential optical path difference (OPD) between the arms of a ground-based interferometer are dry air and water vapor. The influence of dry air, called the piston effect, is almost achromatic in the nearand mid-infrared because the refraction index of dry air is almost constant across this region. The piston mode of turbulence corresponds to the fluctuations of the difference in average phase between the two apertures. In optical interferometry, the piston effect between the two arms of an interferometer induces an erratic movement of the fringes. The power spectrum for the fringe motion follows the classical Kolmogorov spectrum for atmospheric turbulence (Conan et al. 1995), with a typical standard deviation of $20 \mu \mathrm{m}$ rms. In the case of GENIE, this would completely ruin the nulling process because the rms fringe excursion is larger than the fringe spacing itself: the optical axis would therefore see both dark and bright fringes as atmospheric turbulence changes the phase difference between the apertures. Real-time OPD control by means of a fringe tracker is thus mandatory to achieve the required nulling performance, which is related to the rms OPD error by the following relation (Ollivier 1999):

$$
N \simeq \frac{4}{\left(2 \pi \sigma_{\mathrm{OPD}} / \lambda\right)^{2}}
$$


valid for small OPD errors. In order to achieve the nulling ratio of $10^{5}$ required to detect 20-zodi disks, the rms OPD error should be less than about $4 \mathrm{~nm}$, which is one to two orders of magnitude smaller than the performance achieved by current fringe tracking facilities.

Unlike dry air, water vapor is highly dispersive and adds wavelength dependence to OPD fluctuations as the column density of water vapor randomly varies above the two telescopes. This has two consequences on the nulling interferometer, as illustrated in Figure 2:

- Inter-band dispersion. This is the dispersion between the wavelength at which OPD control is performed and the wavelength at which the observation is done. If OPD control is done in the $\mathrm{H}$ band and the observation in the L' band, the typical standard deviation of dispersion-induced OPD is about $0.4 \mu \mathrm{m}$ RMS.

- Intra-band dispersion. This is the dispersion inside the observation waveband itself: because of water-vapor dispersion, the OPD difference between the beams is not the same at the two edges of the observation waveband. The typical rms OPD difference between the two edges of the L' band (3.5 and $4.1 \mu \mathrm{m})$ is about $0.2 \mu \mathrm{m}$.

Both inter-band and intra-band dispersion need to be corrected in real-time in order to maintain a deep and stable nulling of the stellar light. Since dispersion control is not performed by the VLTI, a dedicated corrector will be included inside GENIE.

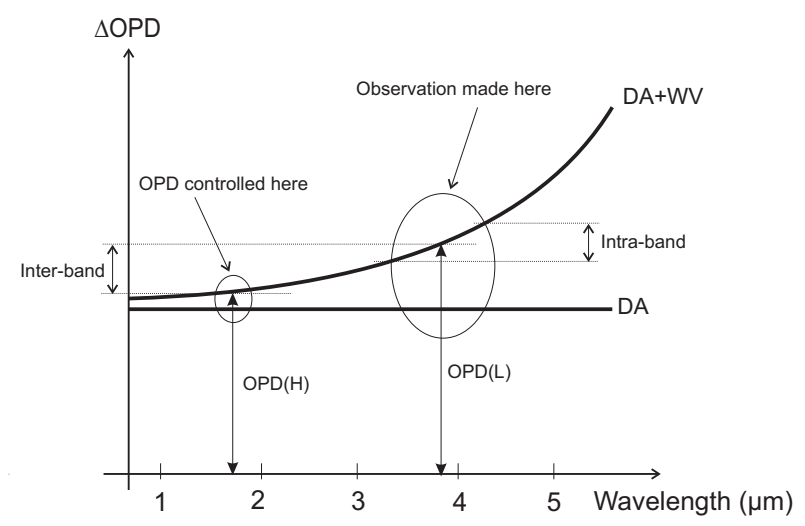

Fig. 2. Illustration of inter-band and intra-band dispersion: the flat solid line is the rms differential OPD expected for only Dry Air, while the solid curve is the rms differential $\mathrm{OPD}$ in the presence of Water Vapor. Fringe tracking is supposed to be performed in the $\mathrm{H}$ band.

The two effects discussed so far are related to the difference in the average phase across the two pupils. Another type of phase error is the wavefront error 
across a given pupil, produced by local variations of the refraction index of humid air. Different wavefront errors in the two beams would reduce their mutual coherence and thus produce a incoherent halo on top of the coherent flux (which interferes destructively on-axis). In order to get rid of wavefront errors, modal filters (e.g. single-mode fibers) will be introduced in the arms of the interferometer. The influence of modal filters on the nulling performance is discussed in the next paragraph.

\subsection{Effects of intensity errors}

The effect of intensity mismatches between the two beams of the interferometer is to produce an imperfect interference and thus to transmit some of the stellar light that should have been blocked. The main contributor to intensity mismatches between the beams of the interferometer is the fluctuation of the coupling efficiency into the single-mode waveguides. The coupling efficiency $\eta$ relates to wavefront perturbations by the following relation (Ruilier \& Cassaing 2001):

$$
\eta \simeq \eta_{0} \exp \left(-\sigma_{\phi}^{2}\right)
$$

where $\sigma_{\phi}$ is the rms wavefront error expressed in radians and $\eta_{0}$ the coupling efficiency in absence of wavefront aberrations $\left(\eta_{0} \simeq 79 \%\right.$ in the case of an UT with ideal injection conditions). For small phase aberrations $\left(\sigma_{\phi}<2 \mathrm{rad}\right)$, the Strehl ratio is accurately approximated by $\exp \left(-\sigma_{\phi}^{2}\right)$. This is the case for GENIE since the VLTI provides a good wavefront correction through the MACAO adaptive optics system (on UTs) or the STRAP tip-tilt unit (on ATs). In the following discussion, we will separate the wavefront errors into two terms: the tip-tilt error and the higher order errors gathered in the Strehl ratio (excluding tip-tilt). The quality of the wavefront delivered by the VLTI is given in Table 1.

\begin{tabular}{c|ccc}
\hline & $\mathrm{K}$ & $\mathrm{L}$ & $\mathrm{N}$ \\
\hline Tip-tilt $[\mathrm{mas}]$ & 13 & 13 & 13 \\
$\langle S\rangle$ & 0.36 & 0.70 & 0.95 \\
$\sigma_{S}$ & 0.063 & 0.044 & 0.008 \\
\hline
\end{tabular}

Table 1. Simulated rms tip-tilt, mean Strehl ratio (excluding tip-tilt) and rms Strehl ratio for an 8-m Unit Telescope after correction by MACAO, for an integration time of 2 sec (Wihlem \& Gitton 2003). A seeing angle $\alpha=1$ arcsec is assumed.

The rms Strehl and tip-tilt fluctuations induce coupling fluctuations up to about $10 \%$ of the mean coupled intensity. The nulling ratio associated to a relative intensity mismatch $\sigma_{I}$ is given by (Ollivier 1999):

$$
N \simeq 16 / \sigma_{I}^{2},
$$

so that an rms intensity mismatch of $10 \%$ induce a limitation of the nulling ratio of about $10^{3}$, which is not sufficient to detect faint exozodiacal disks. Real-time intensity control is thus needed in order to meet the required nulling performance. 
An additional contributor to intensity fluctuations is scintillation. Scintillation is the effect of rapid intensity fluctuations of a point-like source as a result of the interference of light rays which are diffracted by turbulent cells. The condition for scintillation to take place is that the light propagates through the atmosphere over distances longer than the Fresnel propagation length: $h \sec z \geq r_{0}^{2} / \lambda$, where $h$ is the atmospheric scale height, $z$ the zenithal angular distance and $r_{0}$ the Fried parameter. With $r_{0} \geq 5 \mathrm{~cm}$ at $\lambda=500 \mathrm{~nm}$ and $r_{0}(\lambda) \propto \lambda^{6 / 5}$, we have $r_{0}^{2} / \lambda \geq 80 \mathrm{~km}$ in the L' band, which is much larger than the atmospheric scale height. The condition for scintillation to appear is thus not satisfied in the midinfrared and the effect of scintillation on intensity mismatches can be neglected.

\subsection{Effects of polarization errors}

The polarization errors that GENIE will encounter are of two types: differential phase shift between the two polarization components $s$ and $p$, and differential rotation between the two beams for an individual polarization component. These effects can indeed be translated into OPD and intensity errors for each individual polarization state. But unlike OPD and intensity errors due to turbulence, these errors are assumed to be static or slow functions of time. It is thus expected that a precise tuning of the instrument during its commissioning phase, possibly repeated a few times during the instrument lifetime, could be sufficient to make polarization errors negligible. If fine tuning is not sufficient, these errors could be measured periodically and then taken into account in post-processing by subtracting the associated additional stellar leakage.

\section{Correction of atmospheric effects}

\subsection{General scheme for a real-time control loop}

Figure 3 represents the block-diagram of a general control loop. The input signal that has to be corrected is characterized by its Power Spectral Density (PSD) $S_{\text {in }}$. A sensing unit (SU) measures the signal at a given repetition frequency. A certain amount of noise is produced by the detection: photon noise and detector read-out noise are the major contributions to the PSD of detection noise $\left(N_{\mathrm{SU}}\right)$. The value measured by the sensing unit is then fed into a controller $(\mathrm{K})$, which computes the correction to be applied. This process is supposed to be noise-free. The actuator (A) finally applies the relevant hardware correction to the input signal in order to compensate for the error. The actuation is not perfect; this is the reason why an amount of noise is added to the correction signal. The PSD of this noise source $\left(N_{\mathrm{A}}\right)$ depends on the design of the actuator. It is not expected to be a dominant noise source. The performance of the control loop basically depends on the number of available photons at the sensing unit, which will determine the maximum repetition frequency at the which the sensor can operate and thus the maximum close-loop control bandwidth for the rejection of atmospheric turbulence. 


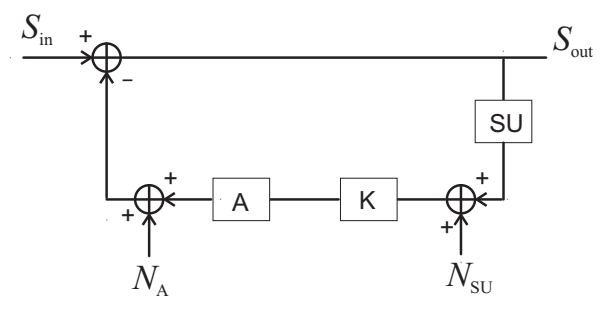

Fig. 3. General scheme for a control loop.

In order to simulate the behaviour of the various control loops foreseen in GENIE, a software simulator for the GENIE instrument, called GENIEsim, has been designed at ESA/ESTEC (Absil et al. 2003).

\subsection{Real-time phase control}

As explained in section 3.1, there are three types of corrections to be applied:

- Piston correction, which can be done at any IR wavelength since piston is achromatic. A first stage of OPD control will be provided by the PRIMA fringe sensing unit and the VLTI main delay lines. However, the correction accuracy (typically $150 \mathrm{~nm} \mathrm{rms}$ on bright targets) is not sufficient for the GENIE purposes. In order to meet the tight specifications on OPD control performance, the control loop should better operate at a wavelength where a large number of photons are available and a high signal-to-noise ratio achievable (i.e., small background emission). Control loop simulations with GENIEsim have shown that the $\mathrm{H}$ band is the most favorable band to perform high-precision OPD control. The correction will most probably be carried out by a fast delay line actuated a piezo-translator operating up to a few tens of $\mathrm{kHz}$.

- Inter-band dispersion correction, which will co-phase the beams at the center of the observation waveband. There are two possible ways to operate the inter-band dispersion control loop: either by measuring directly the phase difference in the observation waveband, or by measuring the phase difference at two different wavelengths (outside the observation waveband) and then extrapolate (or interpolate) the dispersion curve towards the observation waveband to determine the actual phase difference in this band. This latter option is more risky since it relies on a model for the water vapor refraction index, which is of limited precision. Once it has been measured, inter-band dispersion can be corrected by means of a delay line, which can run at a lower frequency than the previous one because dispersion is a smaller effect than piston (its rms is about 100 times less than the rms piston).

- Intra-band dispersion correction, which will co-phase the beams across the 
whole observation waveband. As in the case of inter-band dispersion, the measurement can be made directly in the observation waveband or rely on extrapolation from measurement in other wavebands. In order to meet the specifications, both the first and second order dispersion should be corrected, requiring an actuator made up of two different dispersive materials with variable thickness (e.g. using wedges actuated by piezo translators).

Phase control thus requires phase measurements to be performed at least in two wavebands, and preferably the $\mathrm{H}$ band for piston control and the observation waveband (i.e., L' band) for dispersion control.

\subsection{Real-time intensity control}

The purpose of the intensity control loop is to equalize the intensities in the two beams of the interferometer, but not to stabilize the mean intensities in these two beams. At the output of the intensity control loop, the two beams will thus still fluctuate in intensity, but the two intensities will fluctuate in the same way. In order to preserve the wavefront properties of the beams, the actuator for intensity control should behave as close as possible to a variable density filter. Because of the high actuation frequency required (typically $1 \mathrm{kHz}$ ), a PZT-based actuator is recommended, e.g. a circular shutter with variable diameter.

\section{Preliminary performance estimate for GENIE}

The preliminary nulling performance of GENIE has been estimated in the L' band using the GENIEsim simulator on a typical GENIE target star: a G2 mainsequence star located at $10 \mathrm{pc}$. The performances of the individual control loops and their influence on the achievable nulling ratio are summarized in the following table.

\begin{tabular}{c|ccc}
\hline & $\begin{array}{c}\text { Control loop } \\
\text { frequency }\end{array}$ & $\begin{array}{c}\text { RMS } \\
\text { residual }\end{array}$ & $\begin{array}{c}\text { Equivalent } \\
\text { nulling ratio }\end{array}$ \\
\hline Piston & $20 \mathrm{kHz}$ & $2.8 \mathrm{~nm}$ & $1.8 \times 10^{5}$ \\
Inter-band & $600 \mathrm{~Hz}$ & $1.2 \mathrm{~nm}$ & $1.0 \times 10^{6}$ \\
Intra-band & $600 \mathrm{~Hz}$ & $0.6 \mathrm{~nm}$ & $4.0 \times 10^{6}$ \\
Intensity & $800 \mathrm{~Hz}$ & $1.3 \%$ & $1.1 \times 10^{5}$ \\
\hline Total & - & - & $6.4 \times 10^{4}$ \\
\hline
\end{tabular}

Table 2. Estimation of the GENIE control loop performance and associated nulling ratio obtained with the GENIEsim simulator observing a G2V star at $10 \mathrm{pc}$ in the L' band.

According to these preliminary performance estimates, the GENIE instrument should be capable of maintaining null depths with an accuracy of about $1.6 \times 10^{-5}$ of the initial stellar flux, which should allow to detect exozodiacal disks down to the 30 zodi level. Additional post-calibration techniques are expected to allow a 
slight improvement of this performance and thus to reach the goal 20-zodi level on this typical GENIE target (G2V at $10 \mathrm{pc})$.

\section{References}

Absil, O. \& Bakker, E., 2004, Proc. SPIE, in preparation.

Absil, O., den Hartog, R., Erd, C., Gondoin, P., Kaltenegger, L., Fridlund, M., Rando, N. \& Wilhelm, R., in Toward Other Earths: Darwin/TPF and the Search for Extrasolar Terrestrial Planets, ESA SP-539, p. 317.

Bracewell, R. 1978, Nature, 274, 780.

Conan, J.-M., Rousset, G. \& Madec, P.-Y., 1995, J. Opt. Soc. Am. A, Vol. 12, No. 7, 1559.

Gondoin, P., Absil, O., den Hartog, R., Wilhelm, R., Gitton, P., d'Arcio, L., Fabry, P., Puech, F., Fridlund, M., Schoeller, M., Glindemann, A., Bakker, E., Karlsson, A., Peacock, A., Volonte, S., Paresce, F. \& Richichi, A. 2004, Proc. SPIE, in preparation. Ollivier, M. 1999, PhD thesis, University of Paris XI.

Ruilier, C. \& Cassaing, F., 2001, J. Opt. Soc. Am. A, Vol. 18, No. 1, 143.

Wilhelm, R. \& Gitton, P., 2003, in Toward Other Earths: Darwin/TPF and the Search for Extrasolar Terrestrial Planets, ESA SP-539, p. 659. 